\title{
EFFECT OF UNBROKEN LIGAMENTS ON STRESS CORROSION CRACKING BEHAVIOR OF ALLOY 82H WELDS
}

W. J. Mills and C. M. Brown

\section{NOTICE}

This report was prepared as an account of work sponsored by an agency of the United States Government. Neither the United States Government nor any agency thereof, nor any of their employees, nor any of their contractors, subcontractors or their employees, makes any warranty, express or implied, or assumes any legal liability or responsibility for the accuracy, completeness, or any third party's use or the results of such use of any information, apparatus, product, or process disclosed, or represents that its use would not infringe privately owned rights. Reference herein to any specific commercial product, process, or service by trade name, trademark, manufacturer, or otherwise, does not necessarily constitute or imply its endorsement, recommendation, or favoring by the United States Government or any agency thereof or its contractors or subcontractors. The views and opinions of authors expressed herein do not necessarily state or reflect those of the United States Government or any agency thereof. 


\title{
Effect of Unbroken Ligaments on Stress Corrosion Cracking Behavior of Alloy $82 \mathrm{H}$ Welds
}

\author{
W. J. Mills and C. M. Brown
}

\begin{abstract}
Previously reported stress corrosion cracking (SCC) rates for Alloy $82 \mathrm{H}$ gas-tungsten-arc welds tested in $360^{\circ} \mathrm{C}$ water showed tremendous variability. ${ }^{(1)}$ The excessive data scatter was attributed to the variations in microstructure, mechanical properties and residual stresses that are common in welds. In the current study, however, re-evaluation of the SCC data revealed that the large data scatter was an anomaly due to erroneous crack growth rates inferred from crack mouth opening displacement (CMOD) measurements. Apparently, CMOD measurements provided reasonably accurate SCC rates for some specimens, but grossly overestimated rates in others. The overprediction was associated with large unbroken ligaments that often form in welds in the wake of advancing crack fronts. When ligaments were particularly large, they prevented crack mouth deflection, so apparent crack incubation times (ie, period of time before crack advance commences) based on CMOD measurements were unrealistically long. During the final stages of testing, ligaments began to separate allowing the crack mouth to open rather quickly. This behavior was interpreted as a rapid crack advance, but it actually reflects the ligament separation rate, not the SCC rate. Revised crack growth rates obtained in this study exhibit substantially less scatter than that previously reported. The effects of crack orientation and fatigue flutter loading on SCC rates in $82 \mathrm{H}$ welds are also discussed.
\end{abstract}




\section{INTRODUCTION}

Reference (1) reported that Alloy $82 \mathrm{H}$ welds exhibited tremendous variability in stress corrosion cracking (SCC) behavior in $360^{\circ} \mathrm{C}$ water. Measured crack growth rates (CGRs) bracketed the entire range of rates for Alloy 600 , including both resistant and susceptible heats. The excessive scatter for the welds was attributed to local variations in microstructure, mechanical properties, residual strains and residual stresses that tend to develop in multiple pass welds. It was noted the a single shielded-metal-arc (SMA) Alloy 182 weld tested in BWR hydrogen water chemistry exhibited a similar range of data scatter, ${ }^{(2)}$ thereby suggesting that this level of variability is common in Alloy 600 welds.

Recently reported CGRs for SMA Alloy 182 welds $^{(3-5)}$ suggest that data scatter is less than that reported in Reference (1). In addition, these studies indicated that conventional methods for measuring crack extension tend to be inaccurate for Alloy 600 weld metals. Hence, investigators performed single variable tests such that CGRs could be based on destructive examination measurements, whereby the total amount of crack extension is divided by the total test time. This methodology assumes that crack incubation times are much less than total test times, which appears to be a reasonable assumption for weld metals.

SCC tests reported in Reference (1) used reversing DC electric potential drop (EPD) and LVDT-measured crack mouth opening displacement (CMOD) methods to monitor crack extension. Preliminary findings demonstrated that the EPD method was ineffective for monitoring crack extension in constant-load tests. Apparent crack lengths decreased over time due to the presence of unbroken ligaments in the wake of the advancing crack front and nickel bridging across the crack faces, as these tests were conducted at $150 \mathrm{cc} \mathrm{H}_{2} / \mathrm{kg} \mathrm{H}_{2} \mathrm{O}$ which is well within the nickel regime. In addition, increases in material resistivity during elevated temperature testing can also affect EPD measurements. ${ }^{(6)}$ As a result, reported CGRs were based on CMOD measurements, rather than EPD. Although the CMOD method has been used with reasonable success when monitoring cracking rates in wrought metals, the current reassessment indicates that there was a problem with CMOD-inferred CGRs for some weld specimens. Specifically, the CMOD response for specimens with unusually high rates exhibited a very long crack incubation period, followed by rapid cracking. It is now believed that SCC started much earlier, but unbroken ligaments in the wake of an advancing crack front prevented measurable deflection. During the final stages of testing, the ligaments began to fail which 
caused a substantial increase in CMOD over a short period of time, which was interpreted as a rapid crack growth rate.

Fortunately, all of the data reported in Reference (1) were from single variable tests, so test results could be reanalyzed based on total crack extension, as determined from post-test destructive examination (DE), divided by total exposure time. To determine the influence of unbroken ligaments on SCC behavior, the fracture surface morphology was examined. Revised CGRs were compared with literature data for Alloy 600 and Alloy 182 welds. In addition, the effect of fatigue flutter loading (low amplitude, very high stress ratio) on environmental cracking behavior was evaluated.

\section{EXPERIMENTAL PROCEDURE}

Tests were performed on four different gas-tungsten-arc (GTA) Alloy $82 \mathrm{H}$ welds fabricated by three vendors ( $A, B$ and $C$ ). Compact tension (CT) specimens with a width of 30.5 $\mathrm{mm}$ and nominal thickness of $15.2 \mathrm{~mm}$ were machined from the welds and SCC tested in $360^{\circ} \mathrm{C}$ water with $150 \mathrm{cc} \mathrm{H}_{2} / \mathrm{kg} \mathrm{H}_{2} \mathrm{O}$. Specimens contained $20 \%$ round side grooves to minimize out-of-plane cracking and increase the degree of constraint near the surface. As shown in Figure 1, two specimen orientations were evaluated, including the longitudinal (T-L) orientation where the crack propagates parallel to the welding direction and transverse (T-S) orientation where the crack propagates from the weld root toward the crown.

Testing was performed under constant-load conditions, except for a single test that used fatigue flutter loading with a stress ratio $(R=$ minimum load $\div$ maximum load $)$ of 0.9 , a cyclic frequency of 1 cycle per minute (cpm) and a sawtooth waveform with a $95 \%$ rise time and $5 \%$ fall time.

An in situ LVDT was affixed to the front-face of CT specimens to monitor CMOD throughout the test. As reported in Reference (1), the LVDT output was used to determine SCC incubation times and crack growth rates based on the Saxena-Hudak compliance relationship. ${ }^{(7)}$ Compliance-predicted crack lengths were normalized to post-test measurements of the stress corrosion crack length. The CMOD data from Reference (1) were reanalyzed in this study and the resulting CMOD-inferred CGRs for the weld metal are shown in Figure 2. 
Following each test, specimens were broken apart so the amount of stress corrosion cracking could be measured. To assure that the SCC region was readily apparent, specimens were heat tinted in $538^{\circ} \mathrm{C}$ air for 30 to 60 minutes prior to being fatigued apart. The amount of crack extension $(\triangle \mathrm{a})$ was measured using the ASTM E1820 method using 9 or approximately 25 equally spaced measurements across the fracture face. Average CGRs were calculated by dividing the average crack extension ( $\triangle \mathrm{a}_{\mathrm{AVE}}$ - per ASTM E1820) by total exposure time. Maximum CGRs were determined by dividing the maximum crack extension from the equally spaced crack length measurements $\left(\Delta \mathrm{a}_{\mathrm{MAX}}\right)$ by the total exposure time.

\section{RE-EVALUATION OF CRACK GROWTH RATE DATA GENERATED IN $360^{\circ} \mathrm{C}$ WATER}

The CMOD-inferred CGRs for $82 \mathrm{H}$ welds ${ }^{(1)}$ are plotted in Figure 2 along with literature data for Alloy 600 . It is seen that the $82 \mathrm{H}$ welds exhibit a tremendous amount of scatter, with growth rates varying by almost two orders of magnitude. In fact, the range of CGRs is essentially the same as that for the Alloy 600 database, which includes both annealed and coldworked materials, microstructures with different grain boundary decoration, and yield strengths ranging from 241 to $441 \mathrm{MPa}$.

LVDT-measured CMOD responses for specimens with exceptionally high CGRs (ie, $\mathrm{da} / \mathrm{dt}$ greater than $3 \times 10^{-9} \mathrm{~m} / \mathrm{s}$ ) indicated very long crack incubation times followed by rapid crack growth. As shown in Figures $3 a$ and $3 b$, the LVDT output indicates no evidence of crack extension for the first 81 to 85 days, and then there is a rapid increase in displacement over the next 10 to 20 days. In Reference (1) the rapid increase in CMOD was interpreted as crack growth, which lead to very high CMOD-inferred CGRs. It is now believed that SCC occurred early in these tests, but large unbroken ligaments prevented crack mouth deflection. After extensive amounts of SCC occurred, stress intensities within the ligaments increased and eventually they began to separate, which allowed the crack mouth to open. As a result, the apparent high CGRs inferred from LVDT measurements during the final stages of testing actually reflect ligament separation, not the overall SCC rate. Accordingly, the LVDT-inferred cracking rates grossly overestimated the actual CGRs for these weld specimens. It is noted that LVDT measurements provided reasonably accurate CGRs for some specimens when their CMOD response was not severely impacted by unbroken ligaments. 
Examination of SCC fracture surfaces for $82 \mathrm{H}$ welds reveals a propensity for unbroken ligaments. Figure 4 shows that SCC typically initiates in localized regions and proceeds along favorably oriented dendritic grain boundaries. In some welds, large unbroken ligaments exist well behind the advancing crack front and sometimes even into the fatigue precrack region (Figure 4b). In some cases, ligaments exist between two overlapping, but unconnected, cracks with different elevations. Even when crack extension is rather uniform, unbroken ligaments typically remain well behind the crack tip (Figure 4c). When unbroken ligaments are particularly extensive, the crack mouth is not allowed to open, such that incubation times are greatly overestimated. Once ligaments start to rupture, the crack mouth opens quickly, which then causes CGRs to be overestimated.

Because of CMOD measurement problems due to ligament formation, CGRs must be calculated based on the total amount of crack extension and total test time. In this study, test conditions remained constant throughout each test, so SCC rates could be calculated by this method. Fortunately, SCC of $82 \mathrm{H}$ welds appears to initiate early in the test (ie, incubation times are short), so the total exposure time provides a reasonable representation of the time during which cracking occurred. With one exception, exposure times ranged from 70 to 280 days; hence, incubation times should be much less than test times. While one specimen had an exposure time of only 11 days, its LVDT output indicated that SCC initiated within the first day of testing (this specimen, with a CGR of $1 \times 10^{-9} \mathrm{~m} / \mathrm{s}$, had the highest initial $\mathrm{K}_{1}$ level of $60 \mathrm{MPa} \sqrt{\mathrm{m}}$ ). Moreover, its CGR was consistent with a sister specimen from the same weld that was tested under comparable conditions, but for a much longer time (73 days). In general, there was no systematic difference in DE-inferred CGRs with total exposure times, which also suggests that incubation times tend to be significantly less than exposure times.

Many of the test specimens exhibited extensive SCC, such that $K_{1}$ increased significantly over the course of the test. This could lead to a problem in interpreting the data if CGRs increase dramatically as $K_{1}$ levels become very high. Fortunately, Le Hong et al. ${ }^{(5)}$ showed that CGRs tend to be insensitive to applied $K_{1}$ levels in the high $K_{1}$ regime where the current tests were conducted (i.e., doubling the applied $K_{1}$ causes less than a factor of 2 increase in CGRs). Therefore, the average crack growth rate and mean applied $K_{1}$ level for each specimen are judged to provide an accurate measure of the nominal SCC response. 
Average CGRs for $82 \mathrm{H}$ welds (solid symbols), based on total crack extension divided by exposure time, are plotted in Figure 5 along with the Alloy 600 data (open symbols). Note that additional weld data is provided in Figure 5, because CGRs for both LVDT-instrumented and non-instrumented specimens could be calculated by the re-analysis method. It is seen that the weld data lie in the lower half of the Alloy 600 scatter band. The scatter associated with the four welds represented in this figure is about an order of magnitude.

On average, CGRs for T-L oriented specimens appear to be about half of their T-S counterparts, although there is overlap in the data. This behavior is expected based on consideration of the dendritic grain structure. While the T-L and T-S orientations share a common crack plane, cracks in T-S oriented specimens grow parallel to the columnar grain structure, whereas cracks in T-L oriented specimens grow normal to the columnar grains.

Figures 6 and 7 compare the revised CGRs for $82 \mathrm{H}$ welds with literature data for 182 welds. SCC rates for $82 \mathrm{H}$ welds are seen to be slightly lower than those for Bamford's 182 welds, ${ }^{(4)}$ even though the latter were tested at lower temperatures $\left(320^{\circ}\right.$ and $\left.325^{\circ} \mathrm{C}\right)$. By contrast, Figure 7 shows that maximum CGRs for $82 \mathrm{H}$ welds agree reasonably well with Le Hong's data for 182 welds tested in $350^{\circ} \mathrm{C}$ water. ${ }^{(5)}$ The different responses for the two sets of 182 weld data appear to be related to weld-to-weld variability.

The effect of fatigue flutter loading on environmental cracking behavior is shown in Figure 8. Flutter loading with a stress ratio of 0.9 and a cyclic frequency of $1 \mathrm{cpm}$ did not significantly accelerate CGRs relative to constant-load CGRs, as the average CGR in the flutter test was within the band of CGRs for three sister specimens from T-S oriented Weld "A".

\section{CONCLUDING REMARKS}

Re-evaluation of SCC growth rate data reported in Reference (1) revealed that the large data scatter, where CGRs varied by almost two orders of magnitude, was an anomaly associated with erroneous CGRs inferred from CMOD measurements. While CMOD measurements provided reasonable estimates of CGRs for some weld specimens, they appear to grossly overestimate CGRs in other specimens with large unbroken ligaments. The massive ligaments that sometimes formed in the wake of advancing crack fronts prevented crack mouth 
deflection, so apparent crack incubation times were unrealistically long. After stress corrosion cracks extended considerable distances, the ligaments began to fail due to a significant increase in local stress intensities. Ligament separation during the final stages of testing then allowed the crack mouth to open up over a short period of time, which was erroneously interpreted as a high CGR. Thus, the apparent high CGRs inferred from LVDT measurements during the final stages of testing actually reflect the ligament separation rate, not the SCC rate.

Accurate determination of the extent of SCC in weld specimens requires direct examination of fracture surfaces; hence, CGRs are based on the total amount of SCC divided by total exposure time. Fortunately, crack incubation times in welds subjected to active loading tend to be very short, so the total exposure time can be used to accurately predict CGRs. The judgement that crack incubation times are much less than total exposure times is based on 1) the LVDT output for a specimen with an initial $K_{1}$ of $60 \mathrm{MPa} \sqrt{m}$ indicated that cracking initiated within the first day of testing, and 2) there was no systematic difference in CGRs with varying exposure times.

Revised CGRs for $82 \mathrm{H}$ welds in $360^{\circ} \mathrm{C}$ water indicate that the degree of scatter is substantially less than that reported in Reference (1). The four welds used in this study exhibited a scatter factor of about 10X, which is an order of magnitude less than that previously reported.

Although there is considerable overlap in the data, CGRs for L-T specimens tend to be about half of those for T-S specimens.

Fatigue flutter loading with a stress ratio of 0.9 and a cyclic frequency of $1 \mathrm{cpm}$ did not significantly accelerate cracking rates relative to those obtained under constant-load conditions. 


\section{REFERENCES}

1. C. M. Brown and W. J. Mills, "Effect of Water on Mechanical Properties and Stress Corrosion Behavior of Alloy 600, Alloy 690, EN82H Welds, and EN52 Welds, Corrosion, Vol. 55, 1999, pp. 173-186.

2. L. G. Ljungberg, A. Ortnas, P. Stahle, J. L. Nelson, "Stress Corrosion Cracking Initiation in Alloys 600 and 182," Sixth International Symposium on Environmental Degradation of Materials in Nuclear Power Systems-Water Reactors, TMS, 1993, pp. 379-384.

3. W. H. Bamford, J. P. Foster and R. J. Pathania, "An Investigation of Alloy 182 Stress Corrosion Cracking in Simulated PWR Environment," Ninth International Symposium on Environmental Degradation of Materials in Nuclear Power Systems-Water Reactors, TMS, 1999, pp. 279-294.

4. W. H. Bamford, J. P. Foster, K. R. Hsu, L. Tunon-Sanjur and A. Mcllree, "Alloy 182 Weld Crack Growth and Its Impact on Service-Induced Cracking in Operating PWR Plant Piping," Tenth International Symposium on Environmental Degradation of Materials in Nuclear Power Systems-Water Reactors, NACE, 2002 (CD-ROM).

5. S. Le Hong, J. M. Boursier, C. Amzallag and J. Daret, "Measurements of Stress Corrosion Cracking Growth Rates in Weld Alloy 182 in Primary Water of PWR," Tenth International Symposium on Environmental Degradation of Materials in Nuclear Power Systems-Water Reactors, NACE, 2002 (CD-ROM).

6. C. D. Thompson, D. M. Carey and N. L. Perazzo, "Effects of Hydrogen on Electropotential Monitoring of Stress Corrosion Crack Growth," Eighth International Symposium on Environmental Degradation of Materials in Nuclear Power Systems-Water Reactors, ANS, 1997, pp. 366-371.

7. A. Saxena and S. J. Hudak, Jr., "Review and Extension of Compliance Information for Common Crack Growth Specimens," International Journal of Fracture, Vol. 14, 1978, pp. 453-468. 
Figure 1. Orientation of weld specimens.

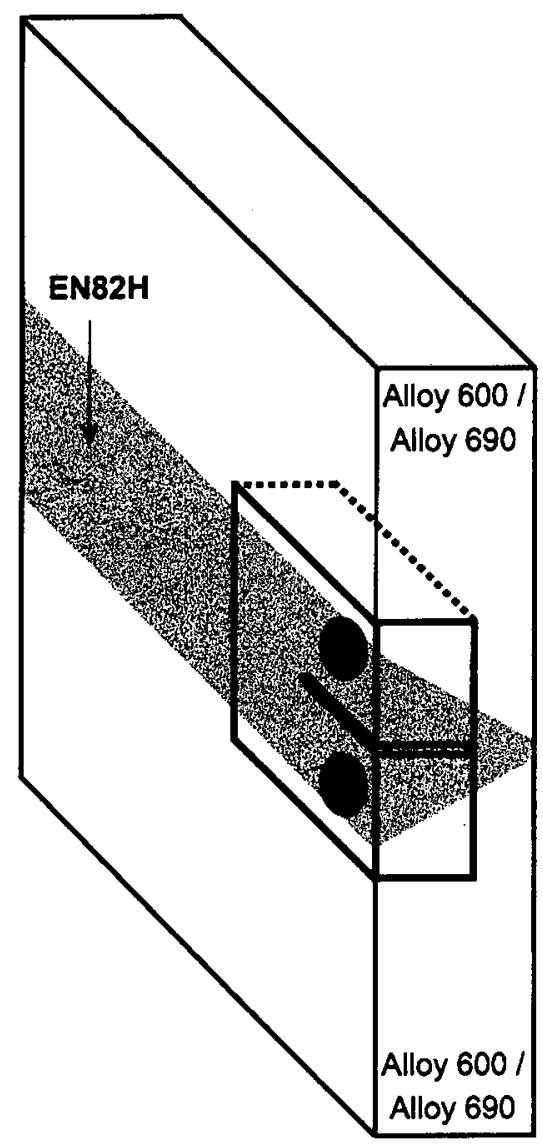

Longitudinal CT

$T-L$

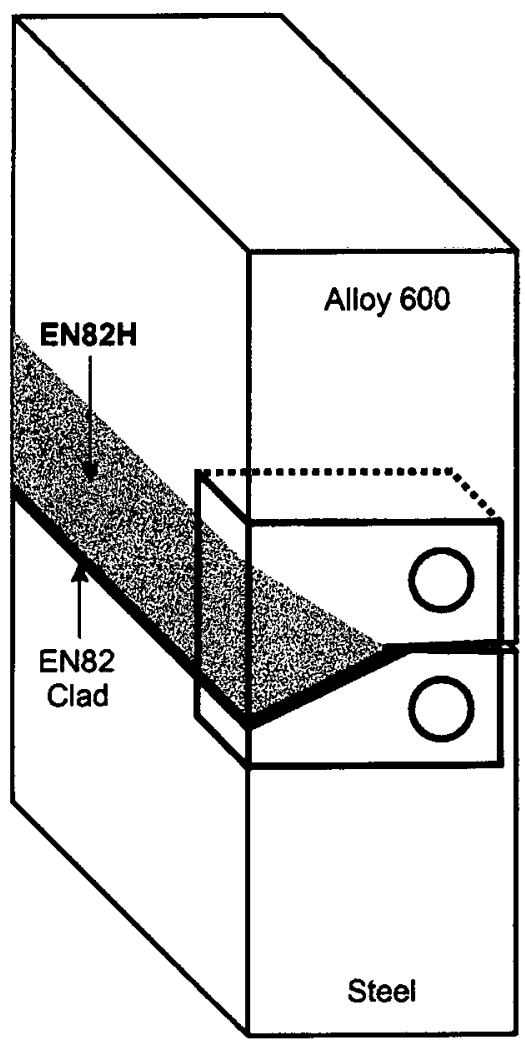

Transverse CT

T-S 


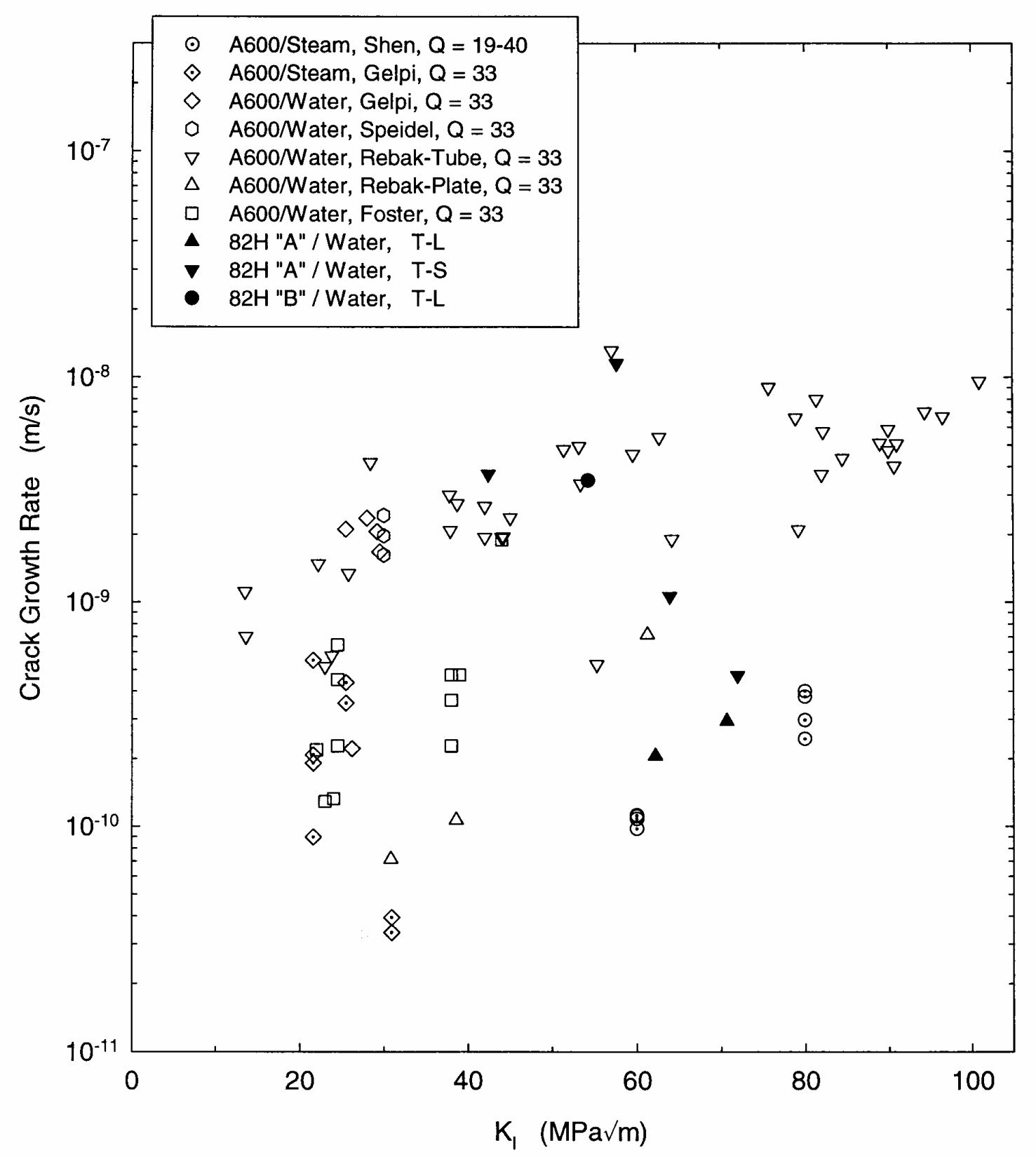

Figure 2 Comparison of CGRs for $82 \mathrm{H}$ welds at $360^{\circ}$, inferred from CMOD measurements, with literature data for Alloy 600 , after References (1). Literature data not generated at $360^{\circ} \mathrm{C}$ were corrected for temperature assuming activation energies ( $Q$ in $\mathrm{kcal} / \mathrm{mol}$ ) provided in legend. 

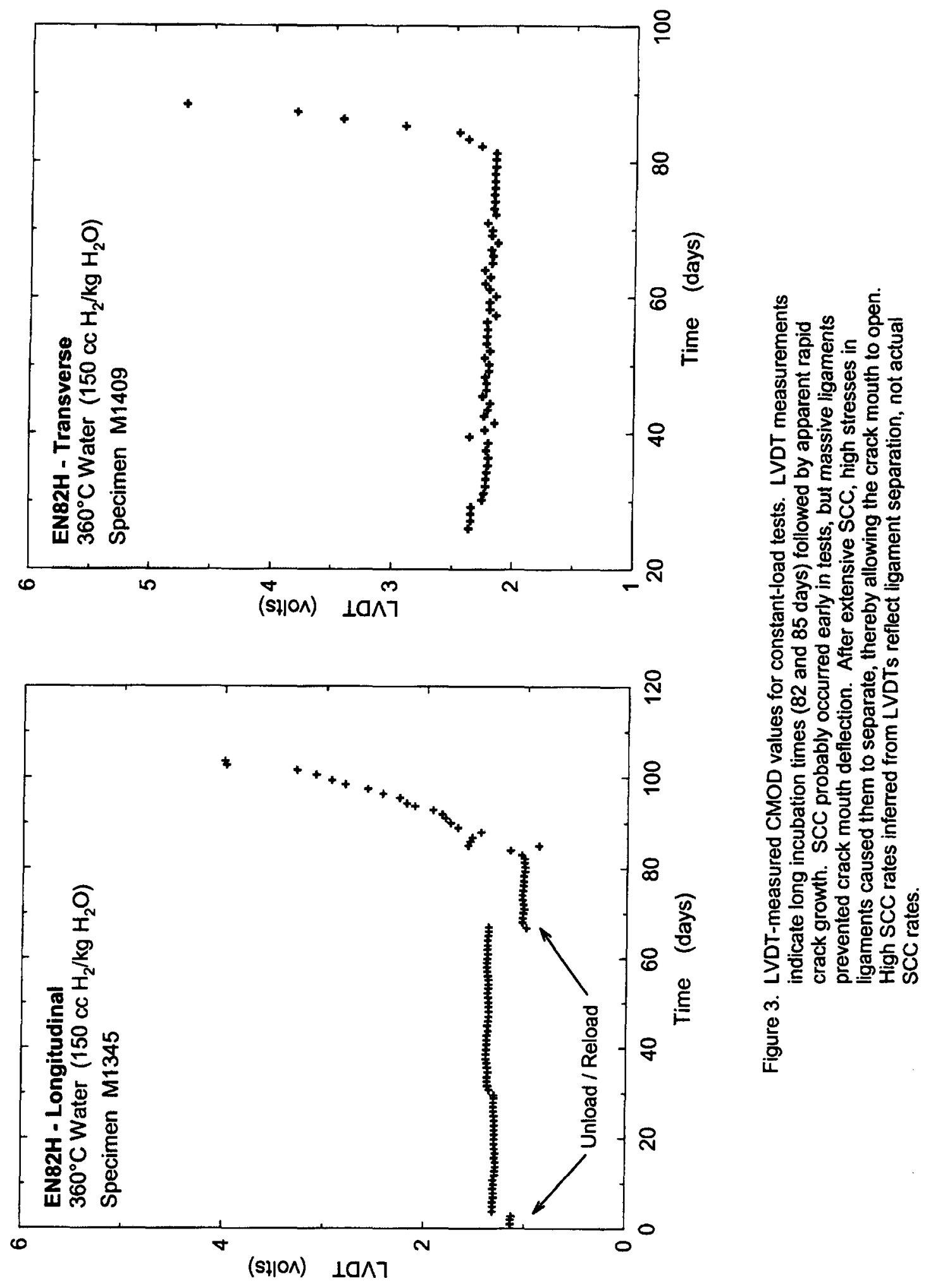


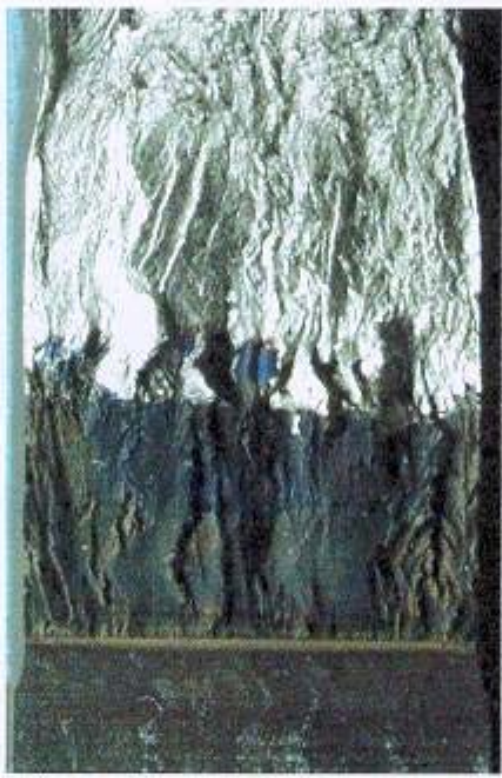

(a)

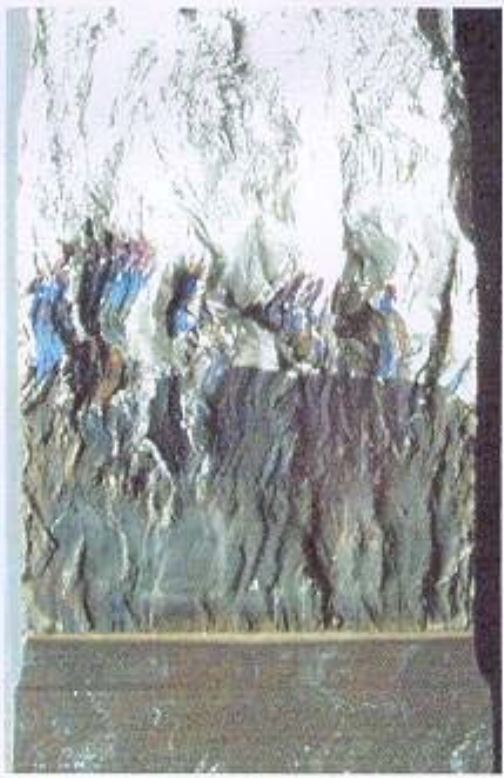

(b)

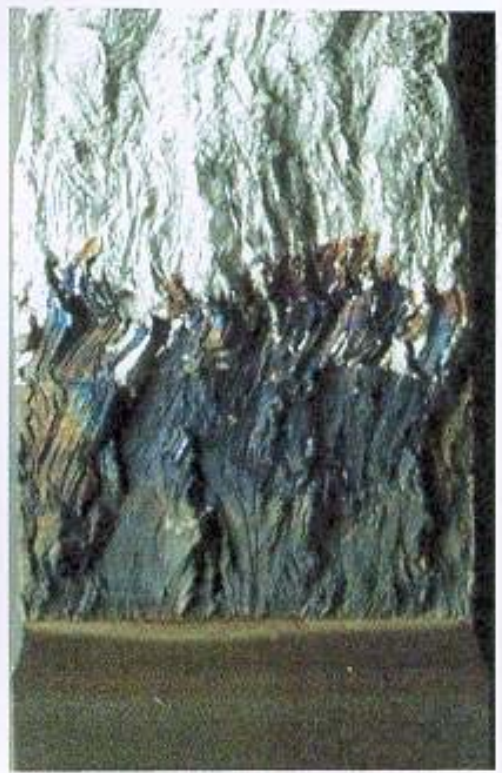

(c)

Figure 4. Fracture surface appearance for Alloy $82 \mathrm{H}$ welds with T-S orientation. Regions from bottom to top: notch, fatigue precrack, stress corrosion crack, post-test fatigue apart (shiny). (a) SCC typically initiates in localized regions and proceeds along favorably oriented dendritic grain boundaries. (b) In some welds large unbroken ligaments (shiny) exist behind advancing crack front. (c) Even when crack extension is rather uniform, unbroken ligaments typically remain well behind crack tip.

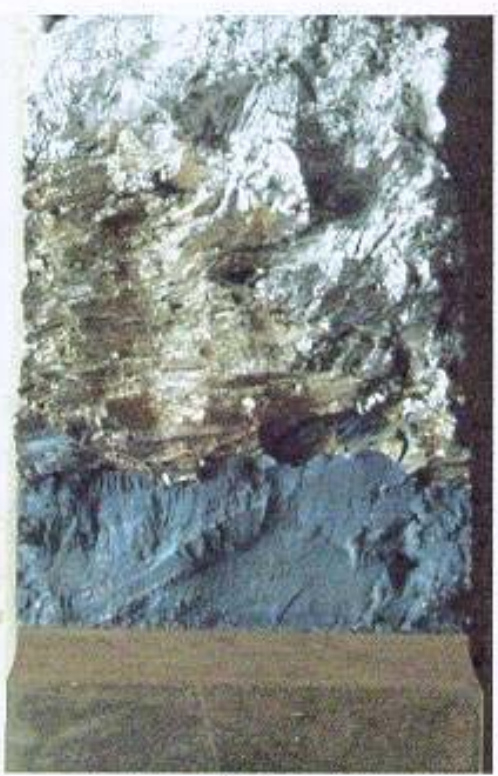

(a)

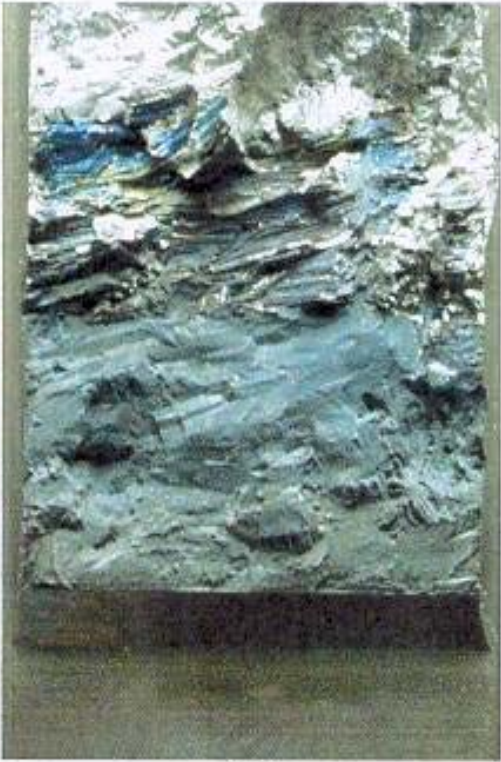

(b)

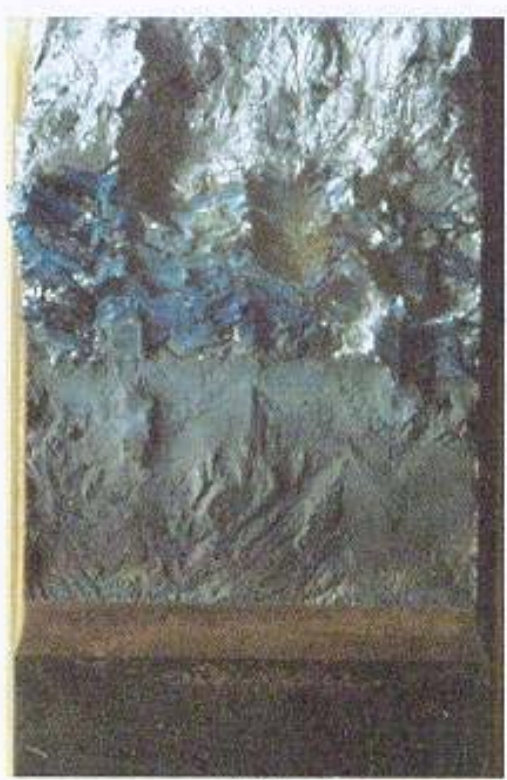

(c)

Figure 5. Fracture surface appearance for Alloy $82 \mathrm{H}$ welds with $\mathrm{T}$-L orientation. (a) Extensive SCC on left side of specimen with massive unbroken ligament on right side. (b) Rather uniform crack extension, except for ligament on far right. (c) Even when crack extension is uniform across entire crack front, unbroken ligaments often remain well behind crack tip. 


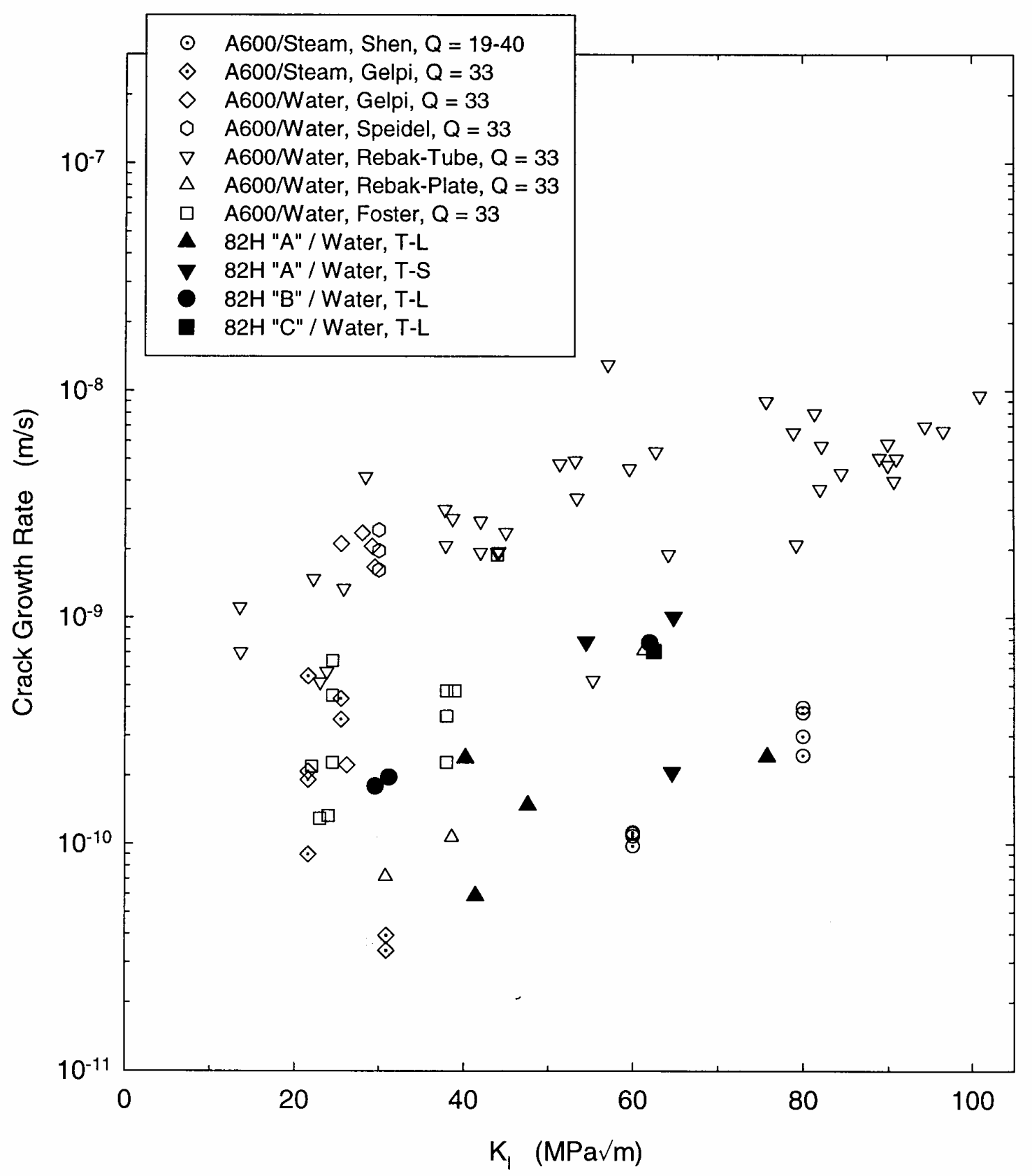

Figure 6. Comparison of SCC rates for $82 \mathrm{H}$ welds at $360^{\circ} \mathrm{C}$ with literature data for Alloy 600 . Crack growth rates for weld metal are based on total crack extension divided by total exposure time. Exposure times (for all but one specimen) ranged from 70 to 280 days; hence, incubation times are much less than test times. Literature data not generated at $360^{\circ} \mathrm{C}$ were corrected for temperature assuming activation energies ( $Q$ in $\mathrm{kcal} / \mathrm{mol}$ ) provided in legend. 


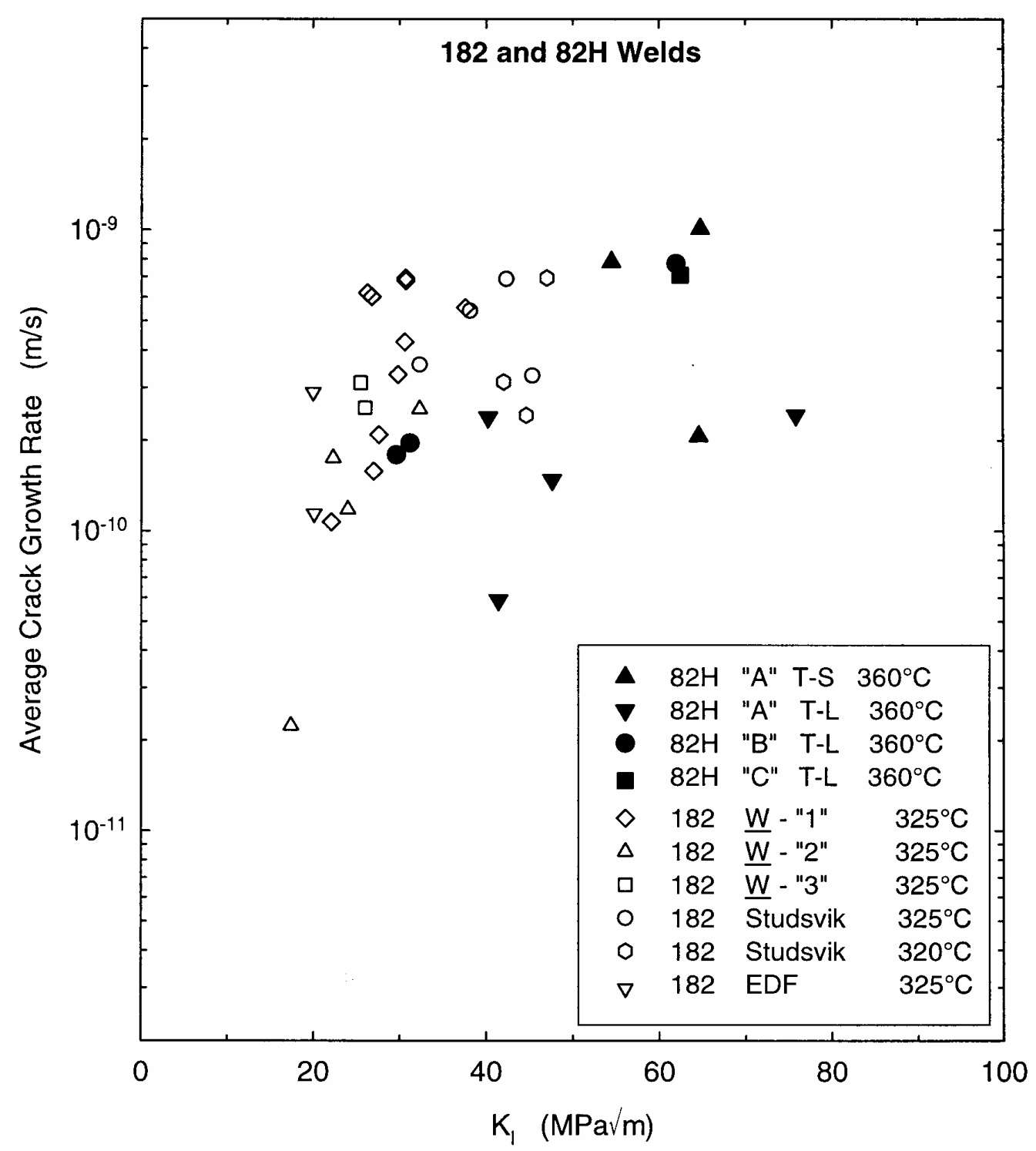

Figure 7. Comparison of average SCC rates for $82 \mathrm{H}$ welds in $360^{\circ} \mathrm{C}$ water with 182 welds in $325^{\circ} \mathrm{C}$ water ${ }^{(4)}$ At intermediate $K_{1}$ levels, crack growth rates for $82 \mathrm{H}$ welds are slightly lower than those for 182 welds, even though the 182 welds were tested at lower temperatures. 


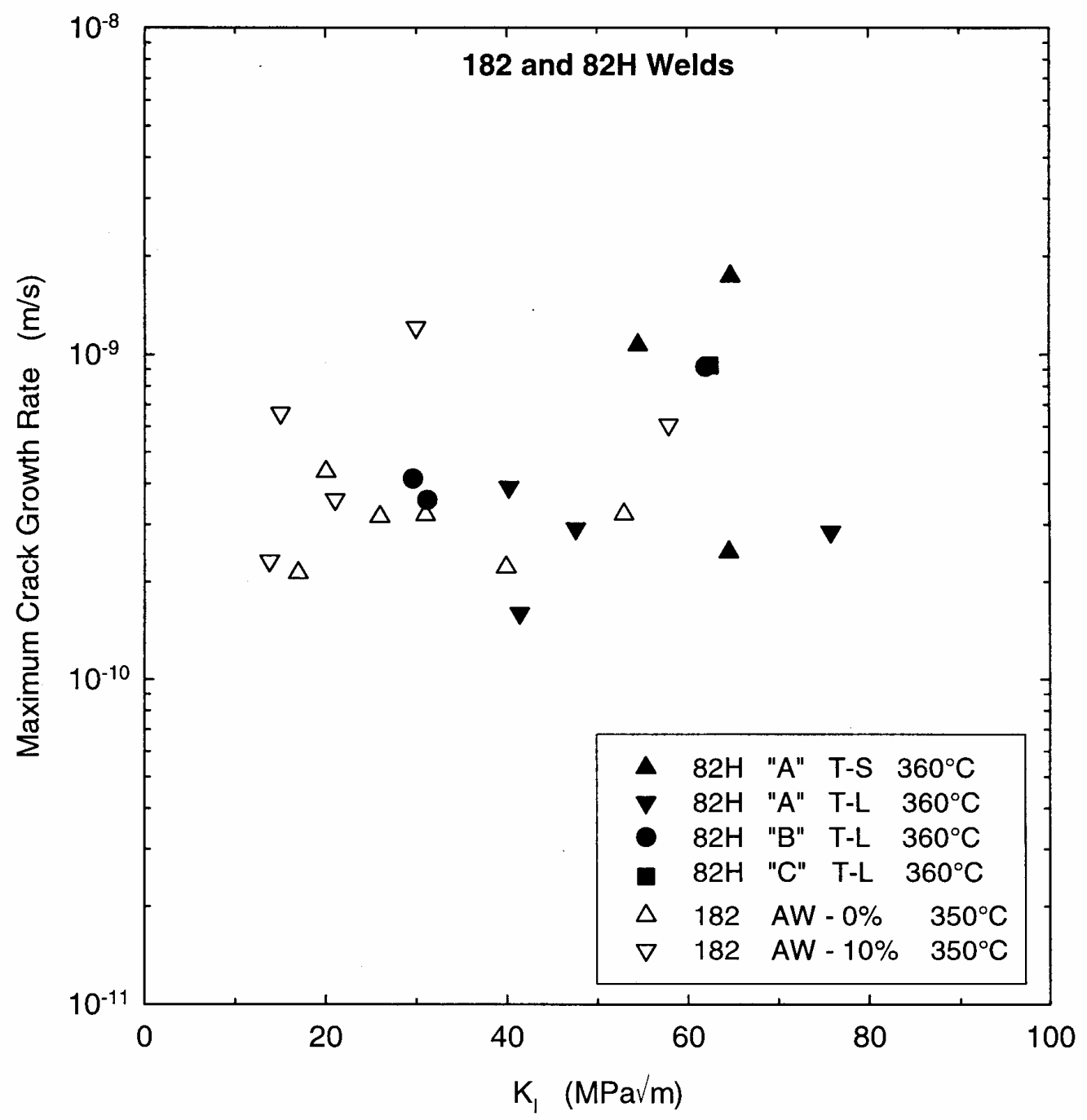

Figure 8. Comparison of maximum SCC rates for $82 \mathrm{H}$ welds in $360^{\circ} \mathrm{C}$ water with 182 welds in $350^{\circ} \mathrm{C}$ water. ${ }^{(5)}$ Crack growth rates for two weld metals agree very well. Alloy 182 welds were tested in both the as-welded and $10 \%$ cold worked conditions. 


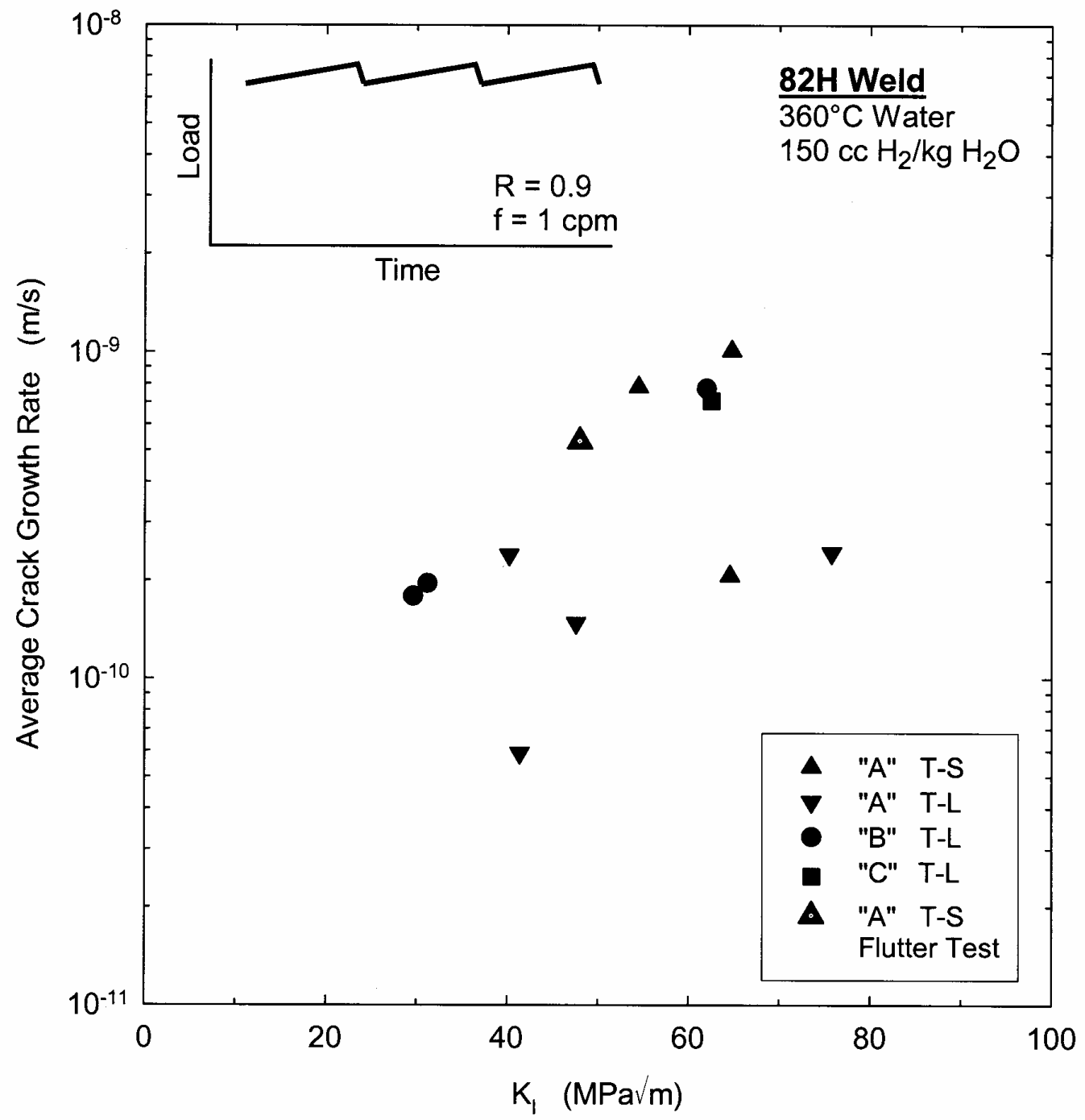

Figure 9. Effect of flutter loading on environmental cracking response. Flutter loading with a stress ratio of 0.9 and cyclic frequency of $1 \mathrm{cpm}$ did not significantly accelerate CGR, relative to CGRs obtained under constant load. 